\title{
ANALYSIS OF FAULT EVENTS IN MVDC ARCHITECTURE
}

By:

\author{
A. Ouroua \\ J.H. Beno \\ R.E. Hebner
}

IEEE Electric Ship Technologies Symposium (ESTS 2009), Baltimore, Maryland, U.S.A., April 20-22, 2009.

PN 325

Center for Electromechanics

The University of Texas at Austin

PRC, Mail Code R7000

Austin, TX 78712

(512) 471-4496

03/10/2009 


\title{
Analysis of Fault Events in MVDC Architecture
}

\author{
A. Ouroua, J. Beno, and R. Hebner \\ Center for Electromechanics \\ The University of Texas at Austin \\ Austin, Texas
}

\begin{abstract}
Effective interruption of load currents under both normal and fault conditions presents major challenges in medium-voltage DC distribution systems. In a highly-integrated DC power system, the preponderance of power converters connecting several loads with power ranging from (W) to (MW) levels rises additional concerns about the effects of a DC fault on other loads connected to the same bus or adjacent buses. Equally critical are the interactions between these power converters during the fault isolation and clearance process. In order to address some of these issues a basic but relevant model has been constructed to study the behavior and effects of DC faults in an MVDC power system for an all-electric ship. Results of the analysis are presented.
\end{abstract}

\section{INTRODUCTION}

In electrical power systems fault analysis allows the calculation of currents and voltages during transient events in order to determine the capacity of various protection devices and assess the dynamic performance of particular architectures, system configurations, or protection schemes. In a large terrestrial power system, the main challenge is to prevent the shutdown of generating units or their disconnection from the power grid during the fault and recovery process in order to avoid potentially catastrophic blackouts. In a highly-integrated power system such as that of a modern electric ship where continuity of service is vital, fault handling mechanisms determine the extent to which power system reconfiguration strategies are possible, with power system reconfiguration being a key property for survivability. Optimum reconfiguration schemes are therefore contingent to an effective fault clearance procedure. Consequently, fault mitigation becomes a major driver in the design of an effective ship DC power system.

To address concerns about fault-handling in a mediumvoltage DC distribution system with several inter-connected generating units, power converters, and DC and $\mathrm{AC}$ loads, we conducted a related study through modeling and simulation. The initial goal of the study is to consider ground and series faults in loads connected to the medium-voltage DC bus (MVDC bus), the low-voltage DC bus (LVDC bus), and the low-voltage AC bus (450 VAC bus), and determine the effects of the disturbances on other system loads and generator. In this paper, we report the initial results of this study using a simple but relevant MVDC power system model.

This work was supported by a grant from the Office of Naval Research
The Matlab/Simulink platform is used in this analysis. A discrete solver and a fixed time step of $1 \mu$ s are appropriate solution method for this problem. The prevalent divergence problems associated with large discontinuities are mitigated by using rate limiters at the output of components that exhibit large changes, mainly power converters. These rates are kept below the $1 \mu$ s resolution whenever possible to reduce errors.

The basic model is described in section II. A detailed description of some component models is given in section III. Fault analysis results and discussions are given in section IV and a conclusion in section $\mathrm{V}$.

\section{BASIC MODEL}

The model used in this analysis represents a simple MVDC architecture. The actual top level Simulink model is shown in Fig. 1. The images on the model components are included to clearly indicate their nature.

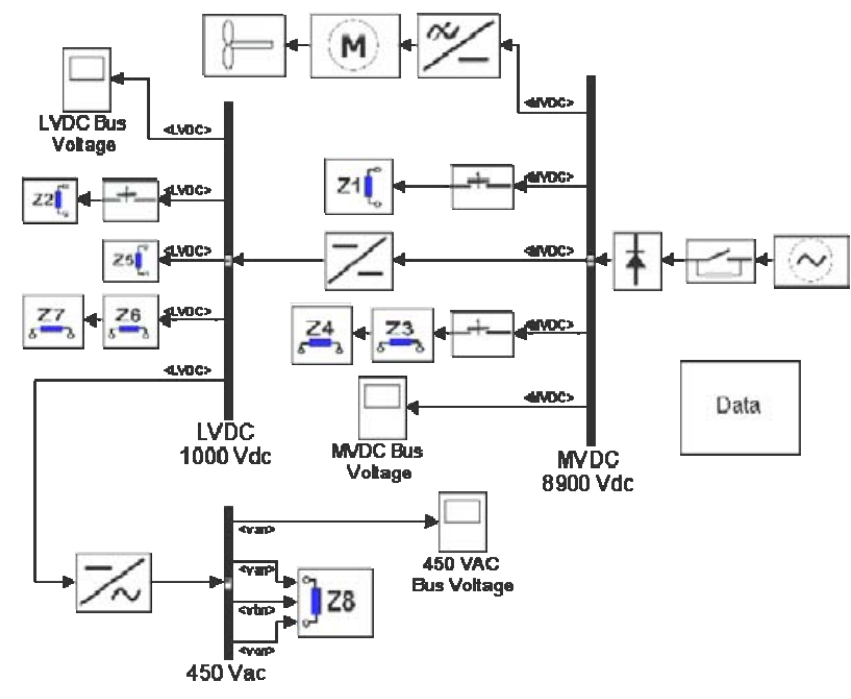

Figure 1. Basic Simulink model for fault analysis.

The model includes a medium-voltage DC bus supplied by a conventional generator and a passive rectifier connected through a 3-phase breaker. The MVDC voltage which is determined by the $6.6 \mathrm{kV}$ generator voltage is $\sim 9 \mathrm{kV}$. A $2-$ MW step-down DC-DC converter supplies a 1000 V DC bus. 
A 3-phase inverter, fed from the low voltage DC bus, supplies a $450 \mathrm{VAC}, 60 \mathrm{~Hz}$, bus. The model includes a propulsion load which consists of a $36 \mathrm{MW} / 120 \mathrm{rpm}$ permanent-magnet synchronous motor driven by a PWM inverter. There are eight additional loads with various power levels in the model. Three DC interrupters, which can represent semiconductor DC breakers [4]-[7], are also included in the model. All power electronics components, except the passive rectifier, are fitted with additional gate signals to selectively turn off the switches when a fault occurs. Finally, we note that a gas turbine was not included in this analysis due to a lack of actual turbine data that are necessary for a credible analysis. In this analysis, the generator is set-up to maintain its output voltage constant through field control. In addition, only a single generator set is considered for simplicity. A gas turbine and a second generator set connected in parallel will be considered in future work.

\section{COMPONENT MODELS}

Appropriate physics models were used to describe the various components in the model. In order to show the level of detail used in this analysis some component models are given explicitly. The propulsion motor is modeled as a synchronous permanent-magnet machine and will not be discussed here. Additional model description and related analysis results can be found in previous publications [1]-[3]. The description of the rectifier model, the generator model, and the step-down converter model follow.

\section{A. Rectifier model}

A 6-pulse passive rectifier was used in this analysis. The model, as depicted in Fig. 2, takes into account the effect of the reactance of the generator to which the rectifier is connected.

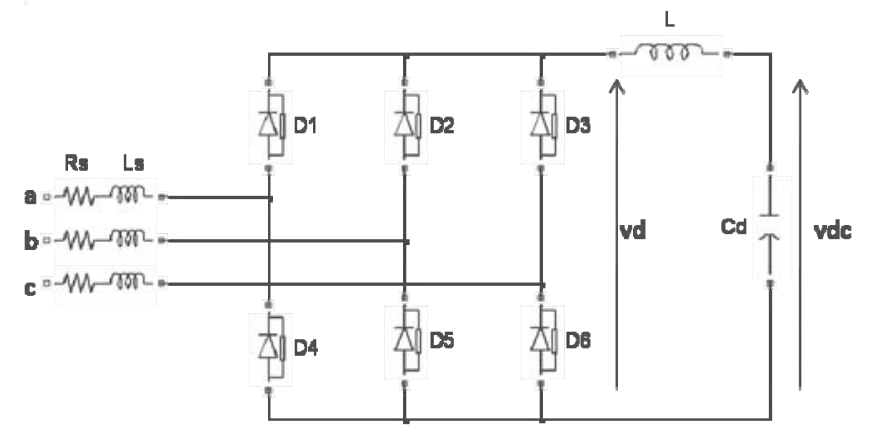

Figure 2. Passive rectifier model.

Model equations, as shown below, are set-up such that the total currents out of each diode and corresponding snubber are calculated along with the output dc voltage. The diodes are represented by small resistances when conducting and very large resistances when blocking. Purely resistive snubbers are used across each diode. An inductor and filter capacitor are included with the rectifier model.

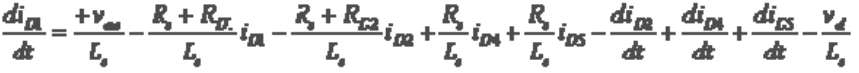

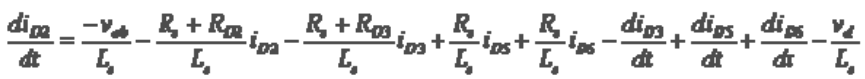

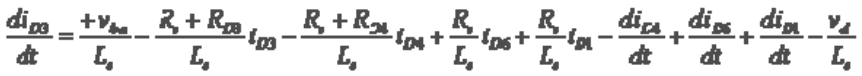

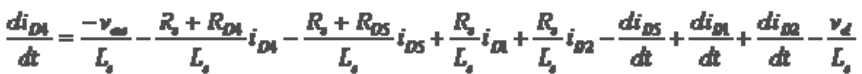

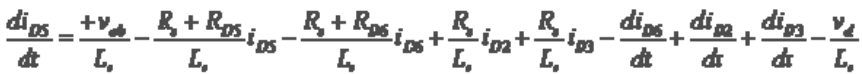

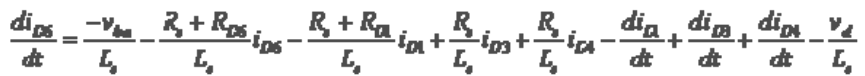

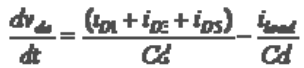

$$
\begin{aligned}
& v_{d}-L \frac{d\left(i_{a}+i_{p 3}+i_{p s}\right)}{d}+v_{d}
\end{aligned}
$$

\section{B. Generator model}

The generator is a wound-field synchronous machine with main specification as follows: 45 MVA, $3600 \mathrm{rpm}, 0.8 \mathrm{PF}$, and $6.6 \mathrm{kV}$. It is modeled with one direct-axis damper winding and one quadratic-axis damper winding. The equivalent corresponding circuits are shown in Fig. 3 . The $p$ operator in the circuit is the derivative $\mathrm{d} / \mathrm{dt}$ and $\lambda \mathrm{d}$ and $\lambda \mathrm{q}$ are linked fluxes in $\mathrm{d}$ - and q-axes. Some values of machine parameters such as inductances are used from previous design studies. When not available, typical values are used.
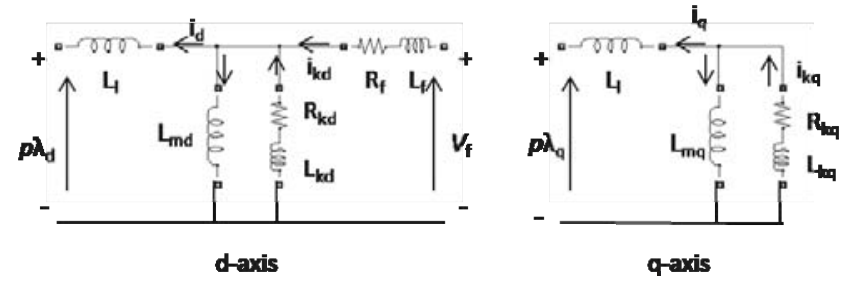

Figure 3. Generator representative circuit with two damper windings.

Model equations in the $\mathrm{dq}$ representation in terms of voltages, currents, and inductances are shown below, along with the electromagnetic torque equation and the dynamic equation. The relations between voltages and linked fluxes are omitted for simplicity but can easily be deduced from Fig.4.

$$
\begin{aligned}
& v_{d}=-R_{a} i_{d}-\left(L_{l}+L_{m d}\right) \frac{d i_{d}}{d t}+L_{m d} \frac{d i_{f}}{d t}+L_{m d} \frac{d i_{k d}}{d t}+\omega_{e}\left(L_{l}+L_{m q}\right) i_{q}-\omega_{c} L_{m q} i_{k q} \\
& v_{q}=-R_{a} i_{q}-\left(L_{l}+L_{m q}\right) \frac{d i_{d}}{d t}+L_{m q} \frac{d i_{k q}}{d t}-\omega_{e}\left(L_{l}+L_{m d}\right) i_{d}+\omega_{g} L_{m d} i_{f}+\omega_{e} L_{m d} i_{k d} \\
& v_{f}=R_{f} i_{f}-\frac{3}{2} L_{m d} \frac{d i_{d}}{d t}+\left(L_{l}+L_{m d}\right) \frac{d i_{f}}{d t}+L_{m d} \frac{d i_{k d}}{d t} \\
& 0=R_{k d} i_{k d}+L_{k d} \frac{d i_{k d}}{d t}+L_{m d}\left(\frac{d i_{f}}{d t}+\frac{d i_{k d}}{d t}-\frac{d i_{d}}{d t}\right) \\
& 0=R_{k q} i_{k q}+L_{k q} \frac{d i_{k q}}{d t}+L_{m q}\left(\frac{d i_{k q}}{d t}-\frac{d i_{q}}{d t}\right) \\
& T_{c}=\frac{3}{2} \frac{P o l e s}{2}\left[\left(L_{m q}-L_{m d}\right) i_{d} i_{q}+L_{m d} i_{f} i_{q}+L_{m d} i_{k d} i_{q}-L_{m q} i_{k q} i_{d}\right] \\
& J \frac{d^{2}\left(\theta_{m}\right)}{d t^{2}}=\left(T_{m}-T_{c}-T_{f r}\right)
\end{aligned}
$$




\section{DC-DC step-down converter model}

The basic topology of the DC-DC step-down converter used in this analysis is shown in Fig. 4. An LC filter is used at the output of the converter. The duty ration of the converter is $\sim 0.11$ which allows a reduction of the voltage down to about 1000 Volts.

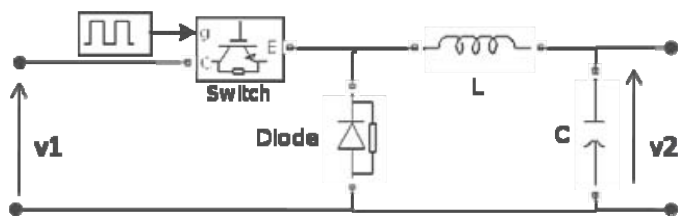

Figure 4. Basic topology of step-down converter.

The switches, which could be IGBTs, are represented by on/off resistors and include resistive snubbers. The diodes are also modeled as on/off resistors when conducting and blocking, as shown in Fig. 5 which represents the model used in the analysis.

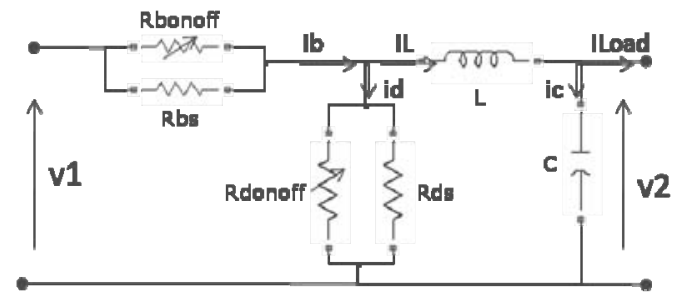

Figure 5. Model of the DC - DC step down converter.

The corresponding model equations are as follows:

$i_{b}=i_{b 1}+i_{b 2}$

$i_{b 1} \cdot R_{\text {tameff }}=i_{82} \cdot R_{t_{6}}$

$v_{d}=v_{1}-i_{b 1} \cdot R_{\text {banaff }}$

$i_{d 1} \cdot R_{\text {danaff }}=i_{d 2} \cdot R_{d s}$

$i_{d}=i_{d 1}+i_{d 2}$

$i_{b}=i_{d}+i_{L}$

$v_{2}=v_{d}-L \frac{d i_{L}}{d t}$

$i_{c}=C \frac{d v_{2}}{d t}$

$i_{L}=i_{c}+i_{\text {Lood }}$

\section{FAULT ANALYSIS}

In this work the analysis of faults in the MVDC power system model is conducted by simulating specific scenarios that are close to actual faults seen in existing power systems or faults that are anticipated in future power systems with novel architectures. Faults are initiated while the power system is functioning normally and supplying power to various loads.

Fig. 6 shows baseline power profiles for all loads with the times they are turned-on during the simulation. Propulsion load is the largest power user and consumes about $6 \mathrm{MW}$ at cruising speed which is assumed here, in relation with the propulsion motor speed, to be about 15 knots. The increasing segment of the propulsion power trace in Fig. 6 corresponds to motor acceleration. Fig. 7 is the propulsion motor speed profile. Some oscillations are present when the motor reaches its operating speed. These do not affect the results of the analyses since the fault scenarios are set-up to occur at time $t$ $=18 \mathrm{~s}$ where motor speed is relatively stable. These oscillations are sensitive to various motor control parameters.

The other load powers vary from the DC load Z2 at $1 \mathrm{MW}$ to the AC load Z8 at $15 \mathrm{~kW}$ as can be seen in Fig. 6.

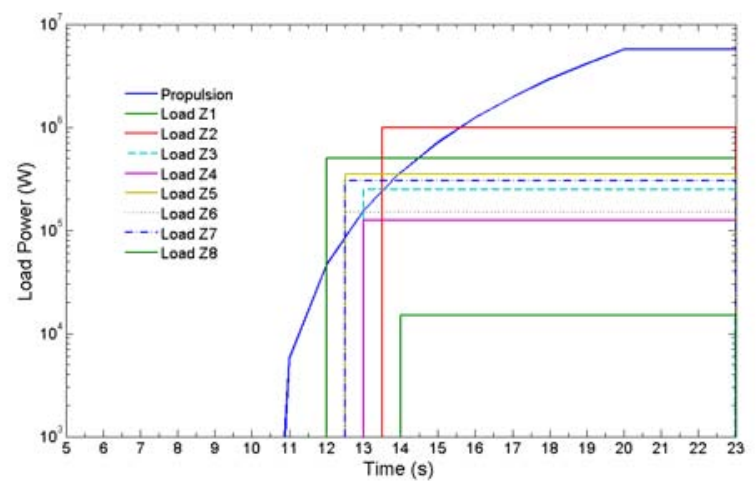

Figure 6. Baseline load profile.

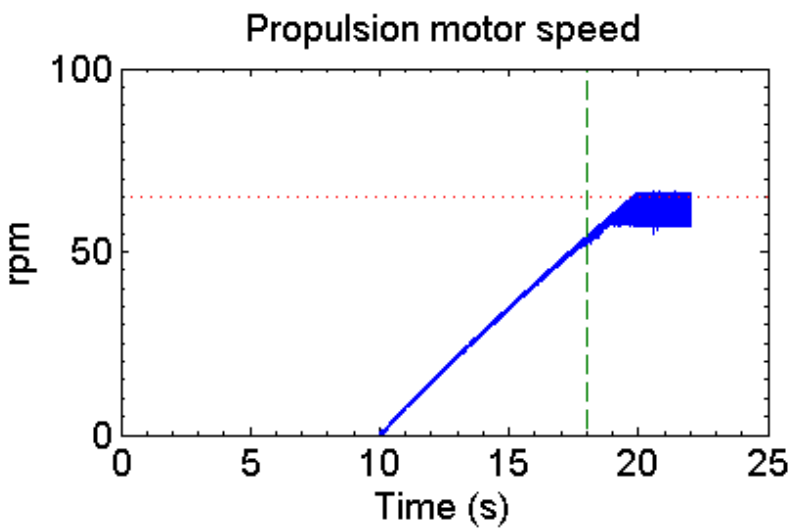

Figure 7. Propulsion motor speed profiile.

The corresponding baseline generator output power is shown in Fig. 8. The three bus voltages at normal operating conditions, i.e. full power consumption and no faults, are shown in Fig. 9. 


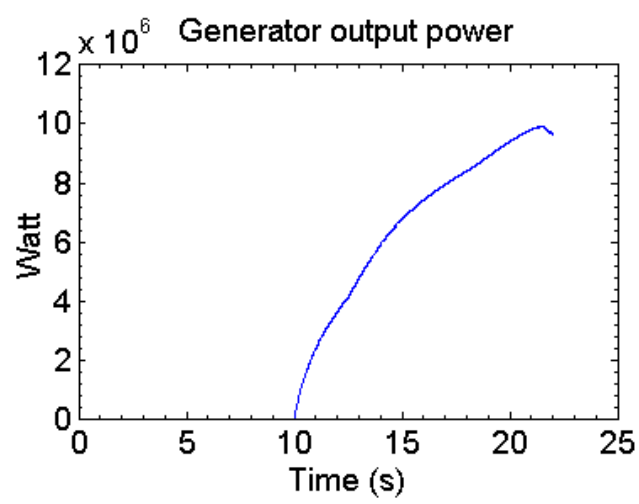

Figure 8. Generator baseline output power.
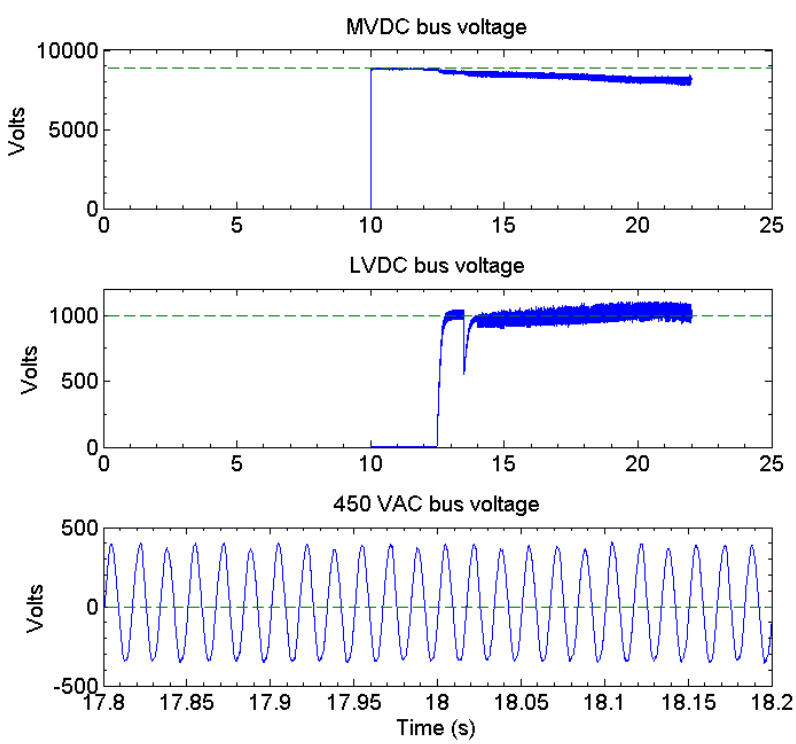

Figure 9. Baseline MVDC, LVDC, and 450 VAC bus voltages.

The sharp drop of voltage in the LVDC bus occurs when power to the $1 \mathrm{MW}$ load (Z2) connected to it is turned-on. This is achieved by closing the DC interrupt 2, depicted in Fig. 1 , which is assumed to be a semiconductor DC breaker and modeled as an on/off resistor with a resistive snubber.

\section{A. Fault in load connected to the MVDC bus}

A ground fault in load $\mathrm{Z} 1$ occurs at time $\mathrm{t}=18 \mathrm{~s}$ while the propulsion motor is accelerating towards cruising speed. All other loads are also supplied with their full rated power when the fault occurs. In this exercise the DC interrupt for load Z1 is not activated, intentionally. Instead, the 3-phase AC breaker is turned-off $5 \mathrm{~ms}$ after the fault is initiated. The ground fault is simulated by setting the load resistance to a very low value, here 1e-3 times the original value, and by controlling the current rise and fall rates to pre-set values. This procedure influences the results but it allows the possibility to change these parameters in order to simulate practical full or partial ground faults or series faults. In addition, it prevents the occurrence of divergences that were mentioned in the introduction while keeping a reasonable degree of fidelity. Time variations of bus voltages during the fault process are shown in Fig. 10.
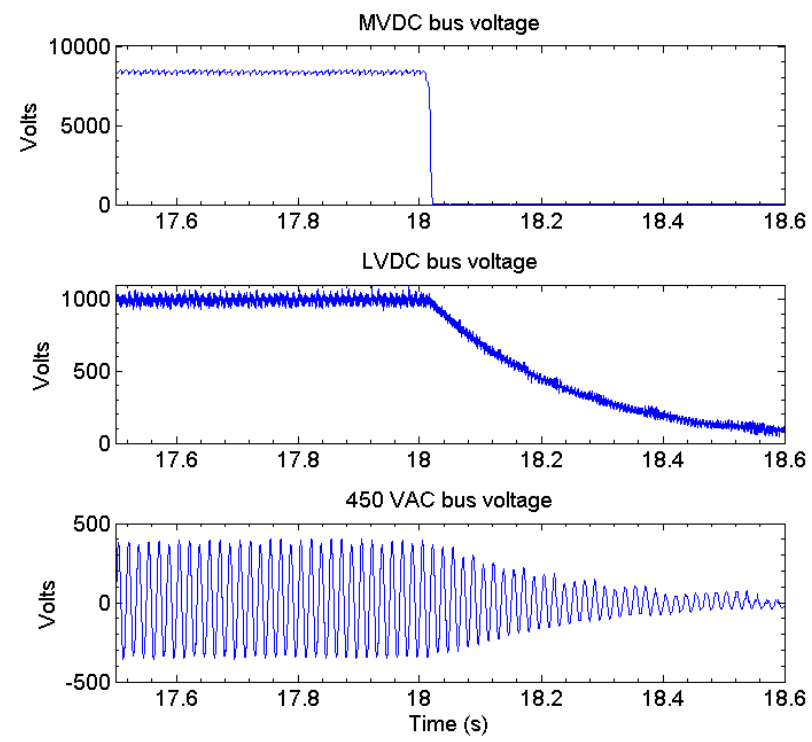

Figure 10. MVDC, LVDC, and 450 VAC bus voltages during fault on load connected to MVDC bus.

In this scenario the main $\mathrm{AC}$ breaker was opened thereby causing all loads to lose power, including the propulsion motor which was accelerating when the fault occurred. Fig. 11 shows the response of the propulsion motor current and voltage during the fault. Equally affected by the fault is the generator current which is included in Fig 11.

For this particular fault scenario no large increases of voltages are seen during the fault and before or after the breaker is opened. However, it is seen that the LVDC and $450 \mathrm{VAC}$ bus voltages decay more slowly than the MVDC bus voltage. The corresponding decay times are strongly influenced by the rectifier, converter, and inverter filter parameters. This observation indicates that the filters used with power electronics need to be designed carefully as they can be used to mitigate DC faults in MVDC architectures. High power filters are usually undesirable components because they are large, occupy valuable space, and reduce efficiency. However, compromises among size, efficiency, and fault tolerance need to be considered during the design of the power system. Analyses such as this one can help with the design process in specifying component parameters and assessing their effects. 

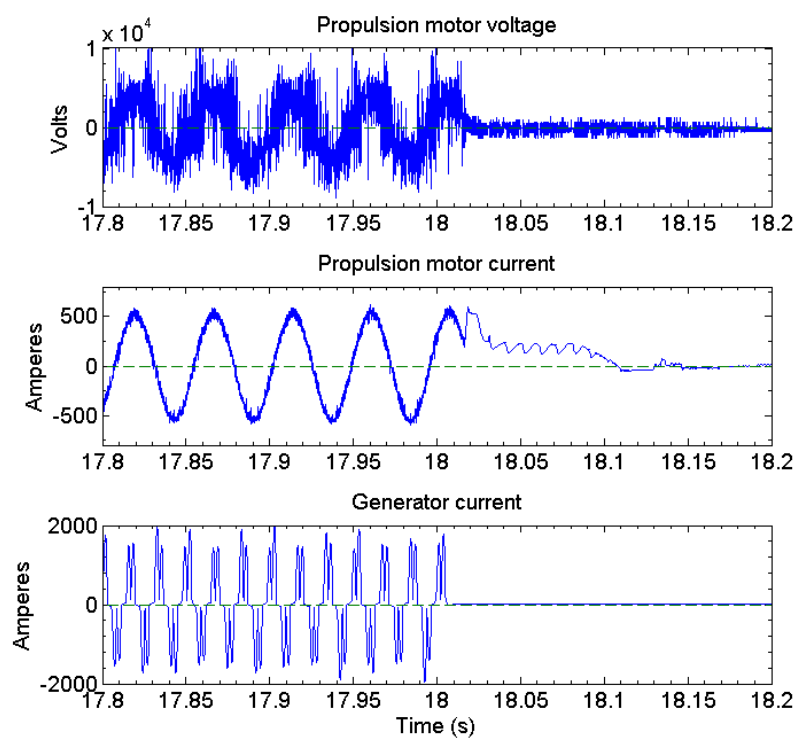

Figure 11. Propulsion motor and generator response to fault in load connected to MVDC bus.

\section{B. Fault in load connected to LVDC bus}

A scenario where a fault occurs in a $1 \mathrm{MW}$ DC load isnow considered. Load Z2 (refer to Fig. 1), connected to the low voltage bus, is shorted to simulate a ground fault at time $t=$ $18 \mathrm{~s}$. During this time all other loads are fully supplied and the propulsion motor is accelerating. The DC interrupt connecting load Z2 to the low voltage DC bus is opened $5 \mathrm{~ms}$ after the ground fault starts. The three bus voltages during the fault are shown in Fig. 12.
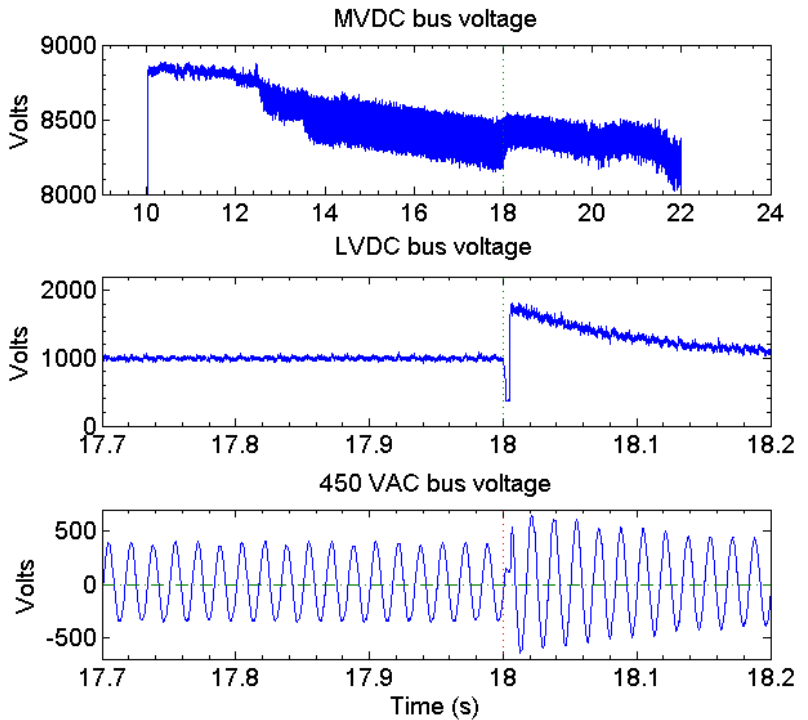

Figure 12. MVDC, LVDC, and $450 \mathrm{VAC}$ bus voltages during fault in $1 \mathrm{MW}$ load connected to LVDC bus.

This fault scenario corresponds to a sharp drop of a $1 \mathrm{MW}$ load from the LVDC bus. As can be seen on Fig. 12, voltages on all buses initially increase then, gradually, decay towards their rated values. Again, converters' filters connected to the supply buses influence the rise and fall of bus voltages during the fault. Fig. 13 shows currents in three different loads connected to the three buses. Since the loads considered here are resistive, they follow the changes in bus voltages to which they are connected. The sharp initial current seen in load Z2 in which the fault occurs, about twice the nominal value, is also influenced by the parameters of the DC interrupter.

Fig. 14 indicates that there are no major disturbances in the propulsion motor and generator when the $1 \mathrm{MW}$ load is dropped off the LVDC bus. The shape of the generator current shown at the bottom of Fig. 14 reflects the highly non-linear nature of the loads the generator is supplying, and this is the case for all scenarios considered here.
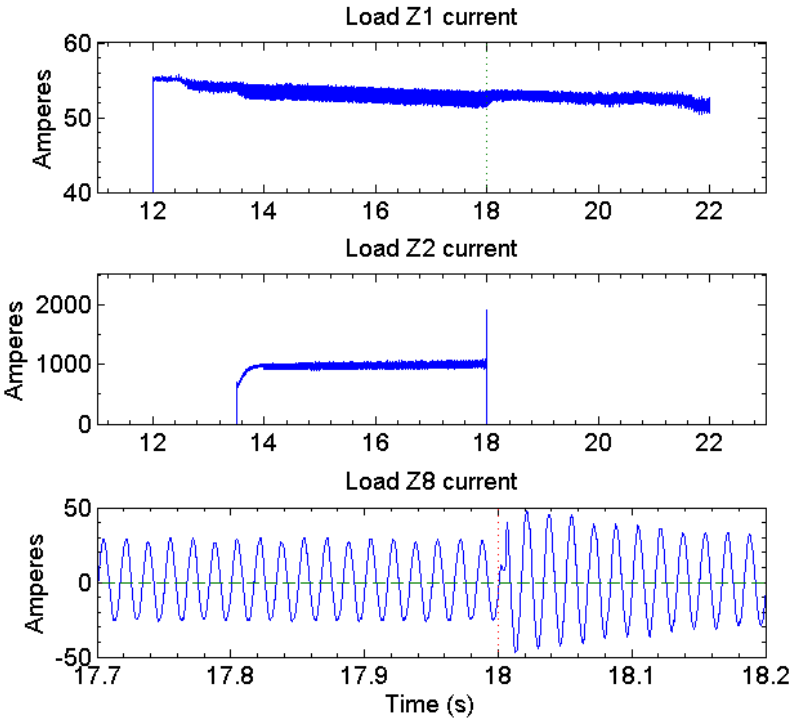

Figure 13. Currents in loads Z1, Z2, and Z8 during fault in LVDC bus.
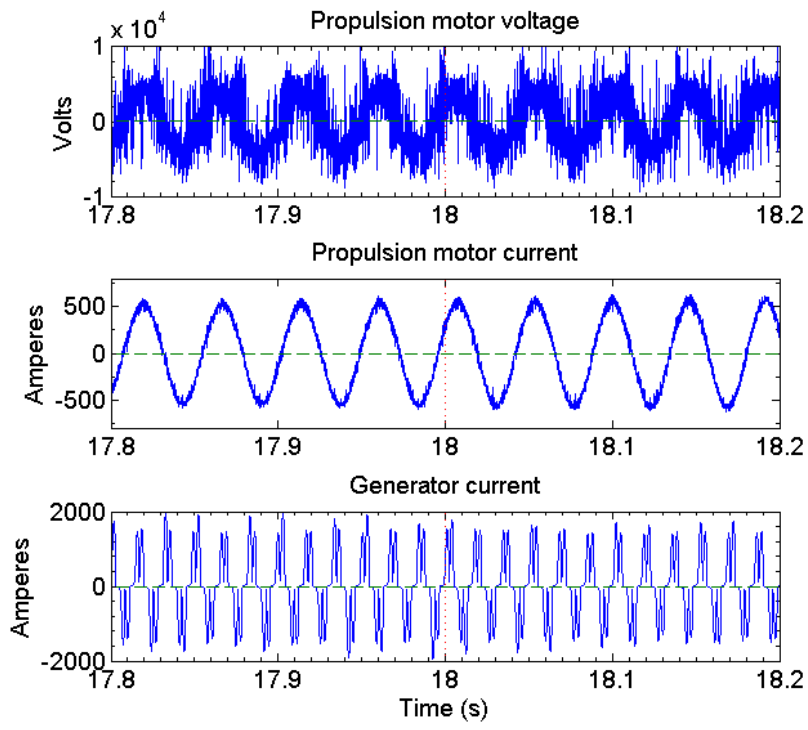

Figure 14. Propulsion motor and generator response to fault in LVDC bus. 
As expected the generator output power, show in Fig. 15, drops by $\sim 1 \mathrm{MW}$, as compared to the baseline power shown on Fig. 8, after the fault occurred.

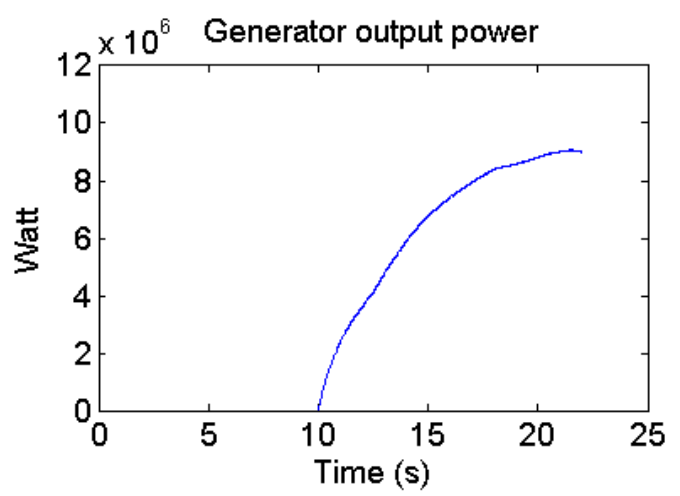

Figure 15. MVDC, LVDC, and 450 VAC bus voltages during fault on load connected to LVDC bus.

\section{Fault in propulsion motor}

In this analysis, machine models are set-up using the dq representation for the case where the system remains balanced at all times. This condition allows a considerable model simplification by the cancellation of the zero-sequence terms. Consideration of unbalanced faults such as line-to-line shorts or single-phase-to-ground faults require the inclusion of the zero-sequence terms or the use of a different reference frame which results in added complexity to machine models. We plan to analyze unbalanced faults in future work.

Two scenarios where a fault occurs in the propulsion motor are now considered. First, a balanced 3-phase ground fault is initiated at time $\mathrm{t}=18 \mathrm{~s}$ then cleared $10 \mathrm{~ms}$ later without any other action. Fig. 16 shows bus voltages during the fault. While there is a small increase of the MVDC bus voltage during the fault, the LVDC and the 450 VAC buses are unaffected.

The PWM motor drive seams to play a positive role in containing the fault within the propulsion motor. However, to ascertain this proposition a detailed look at the currents and voltages in the components of the motor drive, i.e. switches and diodes, is necessary. These data are actually available in this analysis but require additional efforts to be carefully analyzed, a task we plan to undertake in the future.

Currents in loads connected to the three buses are as expected and are shown in Fig. 17. The propulsion motor voltage and current during the fault are shown in Fig. 18.

In this fault set-up the motor voltage is requested to maintain its value at $0 \mathrm{~V}$. The propulsion motor phase current oscillates during the fault as can be seen in the middle graph of Fig. 18. The amplitude of this oscillation is greatly influenced by machine impedance thereby necessitating a good knowledge of machine parameters for meaningful results.
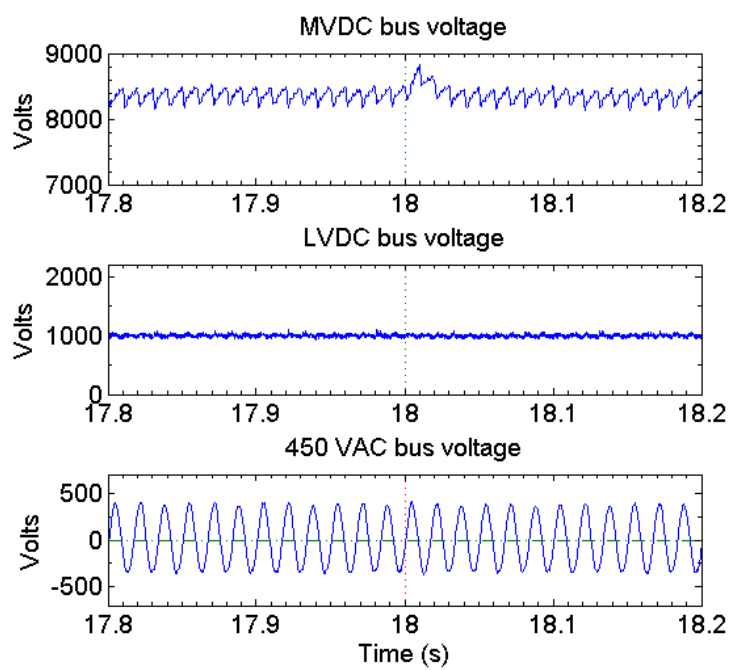

Figure 16. MVDC, LVDC, and 450 VAC bus voltages during fault in propulsion motor.
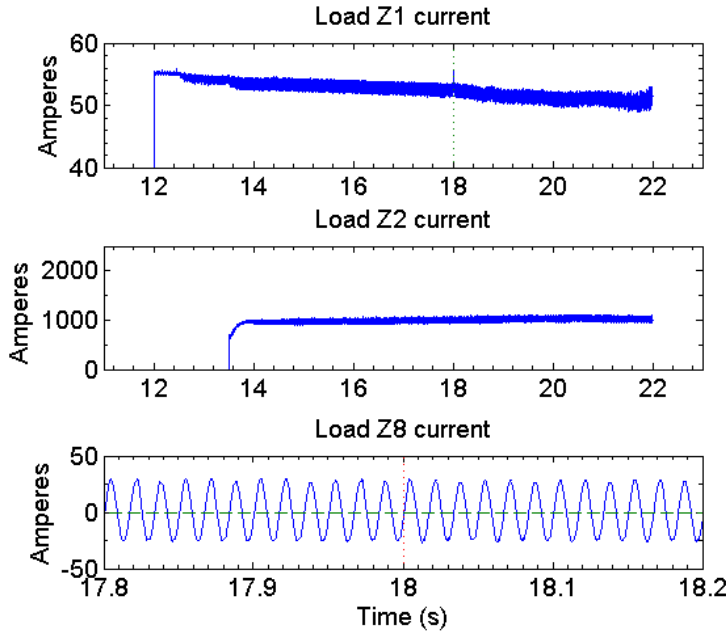

Figure 17. Currents in loads Z1, Z2, and Z8 during fault in propulsion motor.
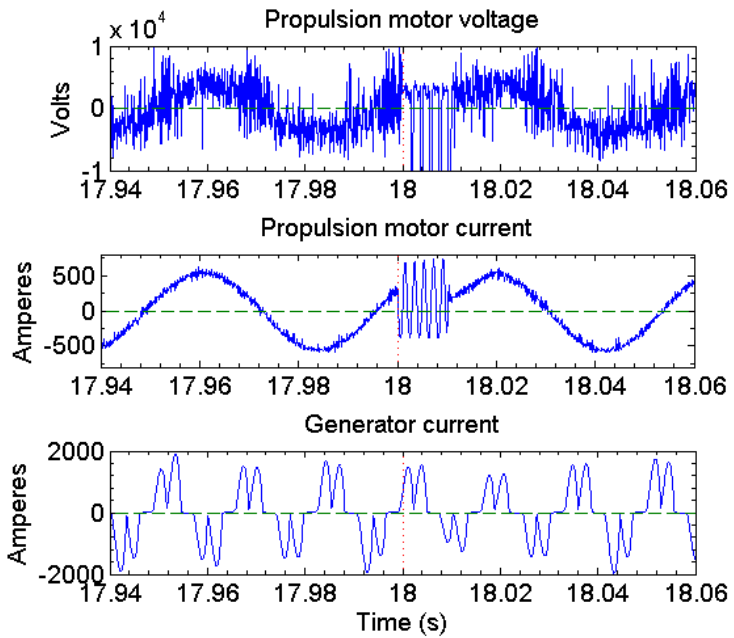

Figure 18. Currents in loads Z1, Z2, and Z8 during fault in propulsion motor. 
In the second scenario a similar balanced-ground fault is applied to the motor windings at time $\mathrm{t}=18 \mathrm{~s}$ but the inverter is shut down $10 \mathrm{~ms}$ after the fault start by requesting that all inverter switches remain in blocking states. In this case the motor loses power after the fault but the other loads are still supplied by their respective buses. Fig. 19 shows motor voltage and current, and generator current for this scenario. The generator current drops considerably, and this was expected since the propulsion motor was consuming about half the power when the fault occurred. This fact is illustrated by the generator output power shown in Fig. 20 .

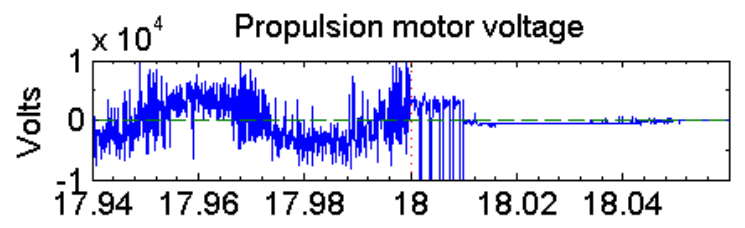

Propulsion motor current
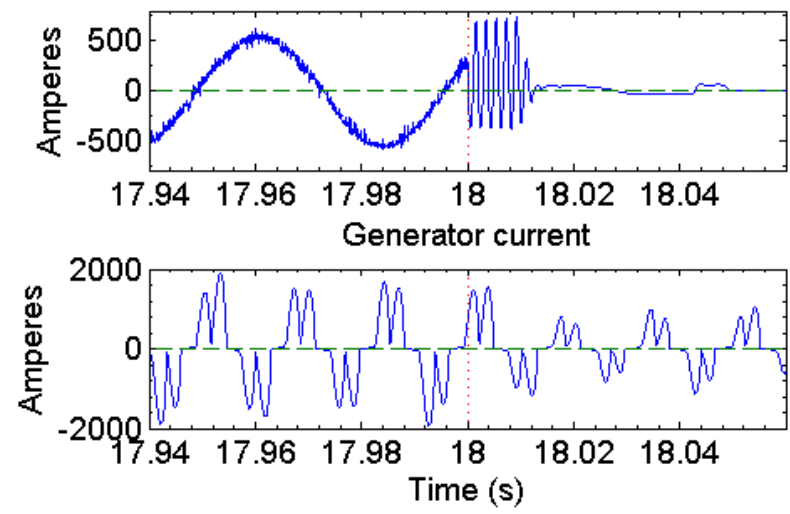

Figure 19. Currents in loads Z1, Z2, and Z8 during fault in propulsion motor with inverter switches in blocking states.

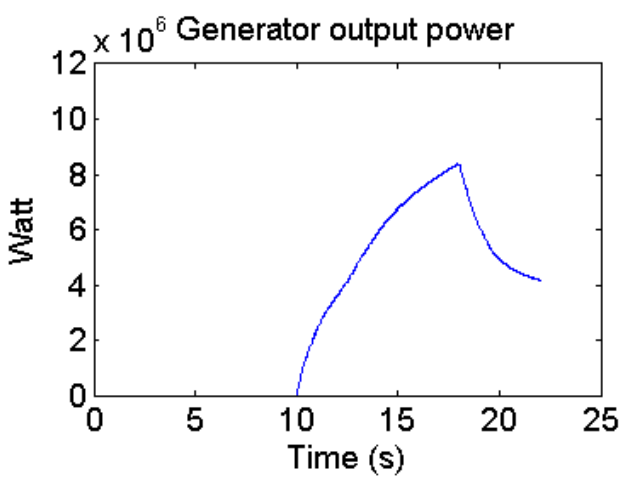

Figure 20. Generator output power during a fault in the propulsion motor with inverter switches operating in blocked state $10 \mathrm{~ms}$ after the fault occurs.

This last exercise demonstrates that power converters can be used to mitigate faults in a medium voltage $\mathrm{DC}$ distribution system if semiconductor power switches with proper voltage and current ratings as well as switching speeds are available. Similar analyses as this one need to be undertaken using actual manufacturer data for advanced semiconductor switches to ascertain their effectiveness in mitigating fault events.

\section{CONCLUSION}

In this analysis a model of a DC distribution system, developed in the Matlab/Simulink modeling environment, was used to analyze fault events in a simplified MVDC power system. Several scenarios where considered to demonstrate the potential usefulness of such an approach. Transient currents and voltages during fault events are calculated in system components of interest using correct physics models for various system components. Knowledge of these parameters can have a considerable impact on the design of an effective MVDC power system. In addition, adequately rated semiconductor power switches used in various power converters can, in principle, be used to mitigate fault events in MVDC architecture.

To further improve the usefulness of this approach, the model needs to be expanded by adding a gas turbine and a second generator set, with different power rating, connected in parallel. Furthermore, the model can be modified to allow the analysis of balanced and unbalanced faults.

\section{REFERENCES}

[1] A. Ouroua, L. Domaschk, and J.H. Beno, "Electric ship power system analysis through modeling and simulation," IEEE Electric Ship Technologies Symposium, July 25-27, 2005, Philadelphia, PA, USA.

[2] S.Z. Vijlee, A. Ouroua, L.N. Domasschk, and J.H. Beno, "Directlycoupled gas turbine permanent magnet generator sets for prime power generation on board electric ships," IEEE Electric Ship Technologies Symposium, May 21-23, 2007, Arlington, VA, USA.

[3] A. Ouroua, J.R. Jackson, J.H. Beno, R.C. Thompson, and E. Schroeder, "Modeling and simulation of electric ships' power system components and their interaction," Summer Computer Simulation Conference, July 15-18, 2007, San Diego, CA, USA.

[4] B. Pauli, G. Mauthe, E. Ruoss, and G. Ecklin, "Development of a high current HVDC circuit breaker with fast fault clearing capability," IEEE Trans. Power Del. Vol.3 , no. 4, pp. 2072-2080, October 1988.

[5] Mesut E. Baran and Nikhil R. Mahajan, "Overcurrent protection on voltage-source-converter-based multiterminal DC distribution system," IEEE Trans. Power Del., Vol. 22, no. 1, pp. 406-412, January 2007.

[6] M. Steurer, K. Frpohlich, W. Holaus, and K. Kaltenegger, "A novel hybrid current-limiting circuit breaker for medium voltage: principle and test results," IEEE Trans. Power Del. Vol. 18, no. 2, pp.460-467, April 2003.

[7] C. Mayer, S. Schroder, and R. W. De Doncker, "Solid-state circuit breakers and fault current limiters for medium-voltage systems having distributed power systems," IEEE Trans. Power Elec., Vol. 19, no. 5, pp. 1333-1340, September 2004. 